\title{
Book report \\ The Little ICU Book of Facts and Formulas
}

Som Sarkar ${ }^{1}$ and Julian Bion ${ }^{2}$

\author{
${ }^{1}$ Specialist Registrar in Anaesthesia and Intensive Care Medicine, University Hospital Birmingham NHS Foundation Trust, \\ Birmingham, B15 2TH, UK \\ 2Professor of Intensive Care Medicine, University Department Anaesthesia and ICM, N5, Queen Elizabeth Hospital, \\ Edgbaston, Birmingham, B15 2TH, UK
}

Corresponding author: Julian Bion, j.f.bion@bham.ac.uk

Published: 19 May 2009

Critical Care 2009, 13:304 (doi:10.1186/cc7764)

This article is online at http://ccforum.com/content/13/3/304

(C) 2009 BioMed Central Ltd

Paul L Marino: The Little ICU Book of Facts and Formulas. Philadelphia: Lippincott Williams and Wilkins, 2009, 800pp., ISBN 978-0-7817-7823-7

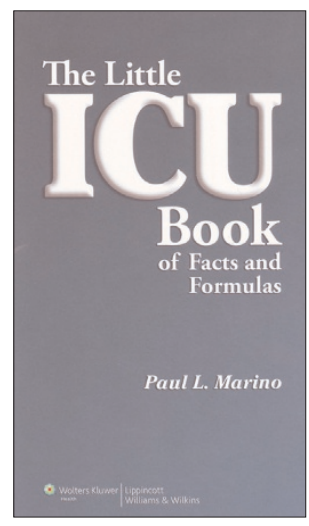

Books now compete with web-based electronic resources, and the challenge for authors and publishers is how to provide accurate and timely information with sufficient depth and breadth to be useful in the workplace. A book of this type therefore has to compromise between size and detail, demonstrate balance in selection of material, and appeal to a globalised world. In many respects, Paul Marino succeeds in this task.

The book is a condensed version of 'The ICU Book' by the same author. Intended as a compact bedside reference, it is presented as 15 chapters, each with up to 5 sub-sections. Chapters cover diverse subjects with an appendix of conversion tables, normal values and formulae. Algorithms are well referenced using current evidence.

However, these strengths are offset by some notable omissions and imbalances. The haemodynamic monitoring section is exclusively based on the pulmonary artery flotation catheter (PAFC), disregarding other forms of monitoring - for example, oesophageal Doppler, $\mathrm{LiDCO}^{\mathrm{TM}}$ or $\mathrm{PiCCO}^{\mathrm{TM}}$. No reference is made to evidence regarding lack of clinical utility of the PAFC. The section on haemodynamic drugs lacks breadth, omitting discussion of epinephrine, vasopressin (mentioned briefly in the septic shock chapter), phosphodiesterase inhibitors or levosimendan. Sodium nitroprusside, however, receives four pages.

The chapter on vascular access mentions the importance of aseptic technique, stating that handwashing, sterile gloves and full barrier precautions are 'recommended' for central venous catheter (CVC) insertion. A simple table mandating such measures would have greater impact, including the use of alcoholic chlorhexidine and a catheter cart facilitating prior preparation. There is no reference to the use of ultrasound guided CVC insertion. Conversely, there is an unnecessary page describing peripheral intravenous cannulation.

The toxicology chapter omits important aspects of selfpoisoning, namely tricyclic antidepressants and drugs of abuse. The chapter on antimicrobial therapy would have been improved by repeating the Surviving Sepsis Campaign recommendations in the septic shock section and by referencing the website, rather than the now outdated 2004 recommendations. Daily review and early termination of antimicrobials should be emphasised.

The sequences of chapters and topic choices are disjointed. Topics including illness severity scoring, morbidity, mortality and treatment withdrawal would have been useful. Non-SI units in many equations make conversions inconveniently necessary for the European reader. The book is positioned as a primer in the basics of North American intensive care medicine. It is not entirely harmonised with European practice and readers may consider alternatives. That said, trainees would find it a handy reference at the ICU workstation.

\section{Competing interests}

The authors delare that they have no competing interests. 